\title{
BMJ Open Effect of particulate matter exposure on patients with COPD and risk reduction through behavioural interventions: the protocol of a prospective panel study
}

\author{
Shinhee Park, ${ }^{1}$ Seung Won Ra, ${ }^{2}$ Sung Yoon Kang, ${ }^{3}$ Hwan-Cheol Kim, ${ }^{4}$ \\ Sei Won Lee (1) ${ }^{5}$
}

To cite: Park S, Ra SW, Kang SY, et al. Effect of particulate matter exposure on patients with COPD and risk reduction through behavioural interventions: the protocol of a prospective panel study. BMJ Open 2020;10:e039394. doi:10.1136/ bmjopen-2020-039394

- Prepublication history for this paper is available online. To view these files, please visit the journal online (http://dx.doi. org/10.1136/bmjopen-2020039394).

H-CK and SWL contributed equally.

Received 16 April 2020 Revised 12 0ctober 2020 Accepted 22 0ctober 2020

Check for updates

(C) Author(s) (or their employer(s)) 2020. Re-use permitted under CC BY-NC. No commercial re-use. See rights and permissions. Published by BMJ.

For numbered affiliations see end of article.

\section{Correspondence to}

Dr Sei Won Lee;

seiwon@amc.seoul.kr and

Dr Hwan-Cheol Kim;

carpediem@inha.ac.kr

\section{ABSTRACT}

Introduction Patients with chronic obstructive pulmonary disease (COPD) are vulnerable to particulate matter (PM) exposure which can increase acute exacerbations and hospitalisation. Interventions to avoid PM exposure are important but evidence-based guidance is lacking. This study aims to assess the impact of PM on lung function, quality of life and exacerbations in patients with COPD using a panel design study; it will also provide evidence for interventional measures to reduce harm from PM exposure.

Methods and analysis A prospective panel study of patients with COPD aged $\geq 40$ years will be conducted. Patients will be required to have a forced expiratory volume in one second $<80 \%$ of the predicted value at enrolment. A total of 120 patients from three different regions will be enrolled, 60 from the metropolitan area, 30 from an industrialised area and 30 from a clean rural area. Clinical outcomes will be assessed through COPD assessment test scores, the St. George's Respiratory Questionnaire for patients with COPD and pulmonary function testing. Indoor and outdoor PM in the patients environments will be measured using gravimetric and light scattering platforms. To estimate the individual dose of PM exposure, a time-activity diary, Geographic Information System and land use regression model will be combined in every season for 1 year. The correlation between PM exposure and the health status of patients with COPD will be evaluated. In addition, 40 patients with the lowest score of life behaviour score to reduce environmental PM exposure will be randomised to a control or intervention group, who will receive in-depth education on riskreducing behaviours.

Ethics and dissemination This study was approved by the Institutional Review Board of each site. The participants received comprehensive information and provided informed consent. The result of this study will be discussed in the form of conference presentations and peer-reviewed publications.

Trial registration number NCT04020237.

\section{INTRODUCTION}

Rapid urbanisation and increased energy consumption around the globe have exposed the human respiratory tract to ambient air
Strength and limitations of this study

- This is the first prospective panel study conducted in Korea to assess the relationship between particulate matter (PM) exposure and symptoms, lung function, quality of life and exacerbations in patients with chronic obstructive pulmonary disease (COPD).

- A total of 120 patients from four different sites will be enrolled from three different areas of Korea to represent real-world air quality, accounting for regional differences in PM exposure.

- With inclusion of the detailed survey of residential environment and life behaviour, their association with PM concentration and clinical parameters can be estimated.

- This study will provide valuable information on the longitudinal relationship between ambient PM exposure and symptoms, lung function and exacerbations of COPD.

- We will identify participants that are at risk of increased PM exposure and interventional education will be implemented.

pollution. Many epidemiological studies show a strong association between ambient air pollutant, that is, particulate matter (PM), and airway diseases. ${ }^{1-3}$ The most affected patients are those with chronic respiratory diseases, such as chronic obstructive pulmonary disease (COPD), the fourth leading cause of death worldwide. ${ }^{4}$ A systematic analysis from the Global Burden of Diseases Study has indicated that ambient air pollution is the second most common cause of morbidity and mortality related to COPD. ${ }^{5}$ Acute exacerbations are associated with increased hospitalisation and mortality in patients with COPD. ${ }^{6}$ A meta-analyses suggest that PM exposure can increase COPD exacerbations, ${ }^{27-9}$ and a recent study showed that PM and other air pollutants increased the risk of severe acute exacerbations. ${ }^{10}$ Air pollutants can also 
aggravate symptoms, quality of life (QOL) and lung function in patients with COPD. ${ }^{11} 12$

Considering the hazardous effect of PM on COPD, interventions to avoid PM exposure are important. Studies of behaviours that can reduce risk have shown that face masks can reduce PM exposure if fitted well and air cleaner use in residences that are near traffic can improve indoor air quality. ${ }^{13-16}$ However, previous studies have focused on either outdoor or indoor exposure to assess the effect of PM exposure on patients with COPD, but neither outdoor nor indoor exposure alone can accurately represent individual PM exposure. In addition, these studies did not provide evidence of the effect of risk-reducing behaviours on symptoms and QOL in patients with COPD.

This study aims to evaluate a panel of 120 patients with COPD to assess the impact of PM on QOL, lung function and acute exacerbations in patients with COPD. It will also aim to intervene to reduce PM exposure in highrisk groups and to assess the effect of this intervention on PM exposure and the clinical parameters of COPD. The details of the study protocol are described.

\section{METHODS AND ANALYSIS}

\section{Study participants and clinical data collection}

The inclusion criteria for the panel are: (1) adults aged $\geq 40$ years of age, (2) patients with COPD, defined as a postbronchodilator forced expiratory volume in one second $\left(\mathrm{FEV}_{1}\right) /$ forced vital capacity $(\mathrm{FVC})<0.7$ and (3) predicted $\mathrm{FEV}_{1}<80 \%$ at enrolment. Patients without respiratory symptoms and those who cannot understand the questionnaires used in the study or air sampler device instructions were excluded. A total of 120 patients from four different sites will be enrolled (figure 1), 60 from the metropolitan area (Seoul, Asan Medical Center and Incheon, Gachon University Gil Medical Center), 30 from an industrialised area (Ulsan, Ulsan University Hospital)

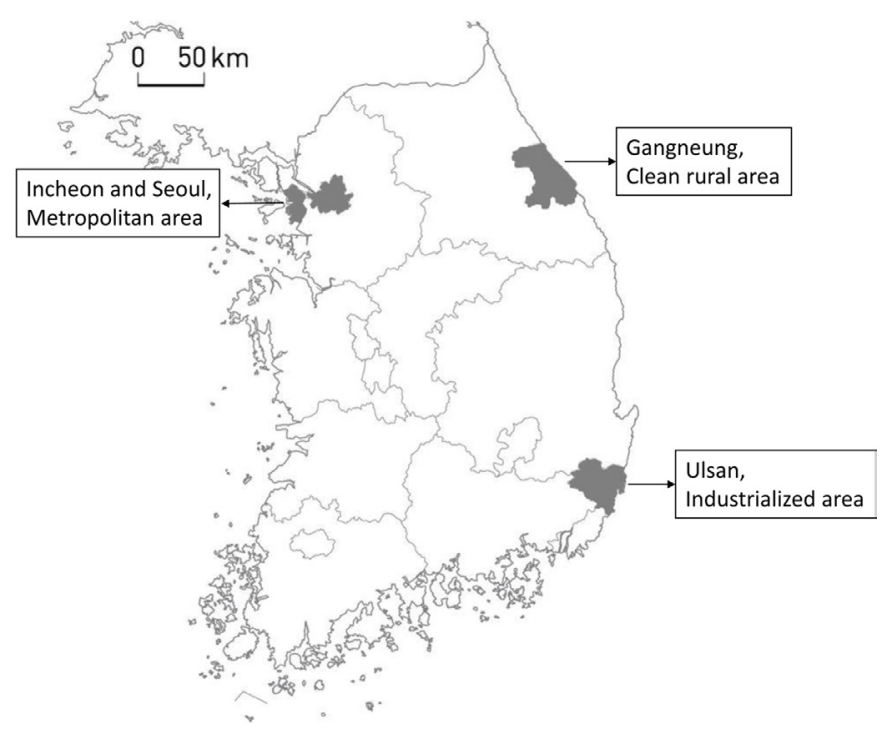

Figure 1 Study sites. and 30 from a clean rural area (Gangwon province, Gangneung Asan Hospital).

Demographic and clinical data, including age, sex, current address, concurrent asthma and history of smoking, use of long-term oxygen therapy will be collected at enrolment. After enrolment, participants will visit hospital every 3 months over the course of a year. To assess QOL and symptoms, the dyspnoea scale, modified Medical Research Council Score and St. George's Respiratory Questionnaire for patients with COPD (SGRQ)-C will be completed at every visit. COPD assessment test (CAT) scores will be obtained on a monthly basis and once a week for a month prior to the hospital visit. Pulmonary function testing, including prebronchodilator and postbronchodilator $\mathrm{FEV}_{1}$ and FVC, will perform at each visit. Clinical parameters, such as acute exacerbations, changes in inhaler use and admissions related to COPD, will also be monitored. During the follow-up period, blood samples will be obtained on two separate occasions when the seasonal PM concentration is high and low. The timeline of data collection is shown in table 1 .

\section{Assessment of the residential environment}

Participants will complete a detailed questionnaire about the indoor and outdoor environment. Questions about the indoor environment include: year of construction and current address; type of housing; number of rooms in the house; type of air conditioning; method of indoor ventilation; presence of an indoor ventilating system; whether the kitchen and living room are separated; recent purchase of new furniture; use of household appliances (air cleaner, humidifier, air conditioner, dehumidifier, gas stove, oil stove, fireplace); use of various chemical agents (mosquito repellent, pesticide, deodorant, kitchen/bathroom disinfectant); whether subjects have recently moved or remodelled; any renovation works; the presence of mould; the presence of flood damage; the presence of pets and type of pet; the type of fuel the family cars use; recent purchase of a car; the presence of a sofa and the material; and the presence of a bed and the materials used to construct the walls of the house.

Questions about the outdoor environment include type of residential location (urban, industrialised or rural); source of air pollutants, distance from the road, traffic volume and whether the participants have moved in the last 2 years and residential address for the last 2 years.

\section{Measurement of individual PM exposure}

Individual PM exposure will be measured using two different methods (the gravimetric method and light scattering) to compare and correct possible errors in the light scattering methods. Indoor PM exposure will be measured every 3 months using a minivolume air sampler (Model KMS-4100, KEMIK Corp., Seongnam, Korea) and two dust spectrometers (11-D, Grimm Technologies and AM520, TSI, Shoreview, USA). When these devices are installed, each participant will receive a portable PM measuring device with Global Positioning System 
Table 1 Timeline of data collection

\begin{tabular}{|c|c|c|c|c|c|c|}
\hline & & Enrolment & $\begin{array}{l}\text { Visit } 1 \text { (3 } \\
\text { months) }\end{array}$ & $\begin{array}{l}\text { Visit } 2 \text { (6 } \\
\text { months) }\end{array}$ & $\begin{array}{l}\text { Visit } 3 \text { (9 } \\
\text { months) }\end{array}$ & $\begin{array}{l}\text { Visit } 4 \text { (12 } \\
\text { months) }\end{array}$ \\
\hline \multirow[t]{3}{*}{ Clinical data } & $\begin{array}{l}\text { CAT } \\
\text { Exacerbations }\end{array}$ & + & + & + & + & + \\
\hline & mMRC, SGRQ-C & + & + & + & + & + \\
\hline & PFT & + & + & + & + & + \\
\hline \multirow{2}{*}{ Environmental assessment } & Activities & & + & + & + & + \\
\hline & Protective behaviour & + & \multicolumn{4}{|c|}{ Education for intervention group at each visit } \\
\hline \multirow[t]{2}{*}{ Indoor PM measurement } & Gravimetric method & & + & + & + & + \\
\hline & Light scattering method & & + & + & + & + \\
\hline Outdoor PM measurement & Air pollution network & \multicolumn{5}{|c|}{$\leftarrow$ continuous monitoring $\rightarrow$} \\
\hline
\end{tabular}

CAT, COPD assessment tool; loT, internet of things; mMRC, modified Medical Research Council; PFT, pulmonary function test; PM, particulate matter; ; SGRQ-C, St. George's Respiratory Questionnaire for patients with COPD.

(Airbeam2 from HabitatMap, Brooklyn, USA), which they will carry on them for 24 hours while logging all their activities in a time-activity diary. A low-cost, sensor-based measurement (CP-16-A5 from Aircok, Seoul, Korea) will also be installed in their homes, which will send measurement data to a server throughout the study period. This device will collect information on PM10, PM2.5, temperature, humidity, volatile organic compounds, formaldehyde and noise level.

Outdoor measurement will be carried out using a similar 'internet of things'-based PM measuring device, which will be installed on enrolment outside the participant's house. Information on weather and air quality (including PM) relating to the residential address and workplace will also be gathered from Air Korea, a national air pollution information system in South Korea (http:// www.airkorea.or.kr).

\section{Statistical estimation for unmeasured PM exposure}

The gaps between individual PM measurements will be estimated with land use regression (LUR) modelling. This approach uses regression coefficients that are calculated using variables such as land use, traffic volume and terrain obtained through a Geographic Information System (GIS) to estimate individual exposure. PM is estimated in a specific geo-coded area according to the residential address using LUR models and data for ambient concentrations of PM10 and PM2.5 will be obtained from Air Korea. LUR modelling we will be used as described in previous studies. ${ }^{12} 17$

\section{Intervention for PM exposure}

A comprehensive questionnaire will be used to evaluate protective behaviour for PM exposure and to identify participants that are at risk of higher indoor PM exposure. This questionnaire was constructed from the list of recommended behaviour developed by the Korean national health department and a list of protective interventions identified through a literature review. ${ }^{14} 15$ 18-20 The questions include several behaviours to minimise PM exposure, such as whether they pay attention to air pollution alerts, use of air filters, frequency of home ventilation while cooking and cleaning, use of face masks during outdoor activities, reduced outdoor activities when PM concentrations are high, etc.

Based on the score obtained from the questionnaire, participants with the lowest score who are at risk of increased PM exposure will be assigned to an intervention group. These subjects will receive 30 min of education at each study visit with the aim of changing their behaviour to reduce PM exposure in everyday life.

\section{Ethical statement and publication}

This study was approved by the Institutional Review Board (IRB) of each site. The IRB of the Asan Medical Center approved the overall concept and execution of the study (2019-0479). The participants received comprehensive information about study and provided informed consent. The result of this study will be discussed in the form of conference presentation and peer-reviewed publications.

\section{Patient and public involvement}

Patients or the public were not involved in the design, or conduct, or reporting, or dissemination plans of our research. 


\section{DISCUSSION}

This is the first prospective panel study conducted in Korea to assess the relationship between PM exposure and symptoms, lung function, QOL and exacerbations in patients with COPD. Individual indoor and outdoor PM exposure data will be collected for 12 months, which will provide information on the longitudinal relationship between PM exposure and symptoms, QOL, lung function and exacerbations. An additional group of patients with low scores for life behaviours that reduce PM exposure will also receive in-depth education to reduce the risk of PM exposure, thereby aiming to provide guidance on reducing PM exposure in everyday life.

There is compelling evidence that shows ambient air pollution can worsen chronic airway diseases. Noh et al reported that increased ambient $\mathrm{O}_{3}$ and PM10 concentrations were associated with increased emergency department visits for the treatment of asthma. ${ }^{21} \mathrm{~A}$ similar trend was observed in patients with COPD. ${ }^{22}$ A meta-analysis showed short-term ambient PM2.5 exposure increases COPD-associated hospitalisations and mortality. ${ }^{9}$ Air pollutants affect symptoms and QOL in patients with COPD. A small panel study conducted in Beijing, China, showed that increasing levels of air pollutants, including PM10 and PM2.5, were associated with increased respiratory symptoms, such as cough, sputum, wheeze and dyspnoea. ${ }^{23}$ Pulmonary function can also be affected by air pollution and one study has shown an increase in lung function in patients with COPD after walking in a park; by contrast, this beneficial effect was attenuated after walking in a street with heavy traffic. ${ }^{24}$

To date, there has been no well-structured cohort study to provide information on individual PM exposure and the relationship with symptoms, lung functions and COPD exacerbations. Therefore, the study described here aims to recruit 120 patients from three different areas of Korea to represent real-world air quality, accounting for regional differences in PM exposure. The participants will be followed up for 1 year, during which time they will provide comprehensive clinical information, including CAT and SGRQ-C, along with longitudinal lung function. Gravimetric and light-scattering platforms will be used to measure indoor and outdoor PM levels and GIS and LUR models will be employed to accurately estimate individual PM exposure.

A small number of studies have shown a long-term longitudinal relationship between ambient PM exposure and respiratory morbidity and mortality. Analysis of patients enrolled in the Multi-Ethnic Study of Atherosclerosis (MESA) has demonstrated that exposure to air pollutants, such as ozone, nitric oxides and PM, is associated with subclinical interstitial lung disease and emphysema. ${ }^{25}{ }^{26}$ Hao et al evaluated the long-term association between ambient ozone, PM2.5 and chronic lower respiratory disease (CLRD) mortality using Bayesian hierarchical spatial Poisson models. ${ }^{27}$ They found that ambient ozone was associated with an increased rate of mortality from CLRD and a similar trend was also observed for
PM2.5, although this difference was not statistically significant. To elucidate the longitudinal relationship between ambient PM exposure and symptoms, lung function and exacerbations of COPD, individual ambient PM level will be measured continuously in the current study. Spatiotemporal exposure models will be used, which were developed from the data of air quality information systems.

As the harmful effect of ambient air pollution on COPD is evident, prophylactic measures should be explored. To date, some research has shown the usefulness of face masks and air cleaners. ${ }^{1428}$ Some reports also suggest water and a high-fibre diet could be helpful, but the evidence is weak. In the current study, expert opinion on how to reduce the risk of PM exposure has been combined to develop a life behaviour score, and 40 patients with the lowest score will be assigned to receive intervention measures or to act as controls. The intervention group will receive a detailed education on how to minimise personal PM exposure in everyday life. Through this intervention, it will be possible to assess the effect of comprehensive lifestyle modification to reduce PM exposure on symptoms, lung function and COPD exacerbations.

In conclusion, the study described here aims to enrol 120 patients with COPD over three different areas in Korea. Symptoms, QOL, lung function and exacerbations will be determined, and indoor and outdoor PM exposure will also be monitored for 1 year. This panel will provide valuable information on regional and lifestyle differences in individual PM exposure and the analysis of estimated PM exposure will improve the understanding of its effect on the life of patients with COPD.

\section{Author affiliations}

${ }^{1}$ Pulmonary, Allergy and Critical Care medicine, Gangneung Asan Hospital, University of Ulsan College of Medicine, Gangneung, Republic of Korea

${ }^{2}$ Department of Internal Medicine, Ulsan University Hospital, University of Ulsan College of Medicine, Ulsan, Republic of Korea

${ }^{3}$ Department of Internal Medicine, Gachon University Gil Medical Center, Incheon, Republic of Korea

${ }^{4}$ Department of Occupational and Environmental Medicine, Inha University College of Medicine, Incheon, Republic of Korea

${ }^{5}$ Department of Pulmonology and Critical Care Medicine, Asan Medical Center, University of Ulsan College of Medicine, Seoul, Republic of Korea

Acknowledgements We would like to thank Bock Hyun Jung and Mi Hye Kim in Gangneung Asan Hospital, Jae Seung Lee, Yeon-Mok Oh and Youngwon Jang in Asan Medical Centre, Sang Pyo Lee in Gachon University Gil Medical Centre, and Geunjoo Na in Inha University College of Medicine for their contribution of this work.

Contributors SWL is the leading investigator of this panel study in collaboration with all other authors (SP, SWR, SYK and H-CK). SWL and H-CK designed the overall study. SWL is responsible for study oversight and coordination. SP and SWL drafted the first version of this manuscript. All authors reviewed the manuscript and approved the final version of the article.

Funding This study was supported by the Research of Korea Centers for Disease Control and Prevention (2019ER671100).

Map disclaimer The depiction of boundaries on this map does not imply the expression of any opinion whatsoever on the part of $B M J$ (or any member of its group) concerning the legal status of any country, territory, jurisdiction or area or of its authorities. This map is provided without any warranty of any kind, either express or implied.

Competing interests None declared. 
Patient and public involvement Patients and/or the public were not involved in the design, or conduct, or reporting, or dissemination plans of this research.

Patient consent for publication Not required.

Provenance and peer review Not commissioned; externally peer reviewed.

Open access This is an open access article distributed in accordance with the Creative Commons Attribution Non Commercial (CC BY-NC 4.0) license, which permits others to distribute, remix, adapt, build upon this work non-commercially, and license their derivative works on different terms, provided the original work is properly cited, appropriate credit is given, any changes made indicated, and the use is non-commercial. See: http://creativecommons.org/licenses/by-nc/4.0/.

ORCID iD

Sei Won Lee http://orcid.org/0000-0003-4814-6730

\section{REFERENCES}

1 Kelly FJ, Fussell JC. Air pollution and airway disease. Clin Exp Allergy 2011;41:1059-71.

2 Moore E, Chatzidiakou L, Kuku M-O, et al. Global associations between air pollutants and chronic obstructive pulmonary disease hospitalizations. A systematic review. Ann Am Thorac Soc 2016;13:1814-27.

3 Guarnieri M, Balmes JR. Outdoor air pollution and asthma. Lancet 2014;383:1581-92.

4 GBD 2017 DALYs and HALE Collaborators. Global, regional, and national disability-adjusted life-years (DALYs) for 359 diseases and injuries and healthy life expectancy (HALE) for 195 countries and territories, 1990-2017: a systematic analysis for the global burden of disease study 2017. Lancet 2018;392:1859-922.

5 Soriano JB, Abajobir AA, Abate KH, et al. Global, regional, and national deaths, prevalence, disability-adjusted life years, and years lived with disability for chronic obstructive pulmonary disease and asthma, 1990-2015: a systematic analysis for the global burden of disease study 2015. Lancet Respir Med 2017;5:691-706.

6 Jinjuvadia C, Jinjuvadia R, Mandapakala C, et al. Trends in outcomes, financial burden, and mortality for acute exacerbation of chronic obstructive pulmonary disease (COPD) in the United States from 2002 to 2010. COPD 2017;14:72-9.

7 Zhu R, Chen Y, Wu S, et al. The relationship between particulate matter (PM10) and hospitalizations and mortality of chronic obstructive pulmonary disease: a meta-analysis. COPD 2013;10:307-15.

8 Atkinson RW, Kang S, Anderson HR, et al. Epidemiological time series studies of PM2.5 and daily mortality and hospital admissions: a systematic review and meta-analysis. Thorax 2014;69:660-5.

9 Li M-H, Fan L-C, Mao B, et al. Short-Term exposure to ambient fine particulate matter increases hospitalizations and mortality in COPD: a systematic review and meta-analysis. Chest 2016;149:447-58.

10 Choi J, Oh JY, Lee YS, et al. Harmful impact of air pollution on severe acute exacerbation of chronic obstructive pulmonary disease: particulate matter is hazardous. Int $J$ Chron Obstruct Pulmon Dis 2018;13:1053-9.

11 Pothirat C, Chaiwong W, Liwsrisakun C, et al. Influence of particulate matter during seasonal smog on quality of life and lung function in patients with chronic obstructive pulmonary disease. Int $J$ Environ Res Public Health 2019;16:106.
12 Lamichhane DK, Leem JH, Kim HC. Associations between ambient particulate matter and nitrogen dioxide and chronic obstructive pulmonary diseases in adults and effect modification by demographic and lifestyle factors. Int $J$ Environ Res Public Health 2018;15:e363.

13 Cox J, Isiugo K, Ryan P, et al. Effectiveness of a portable air cleaner in removing aerosol particles in homes close to highways. Indoor Air 2018;28:818-27.

14 Pacitto A, Amato F, Salmatonidis A, et al. Effectiveness of commercial face masks to reduce personal PM exposure. Sci Total Environ 2019;650:1582-90.

15 Xiong L, Li J, Xia T, et al. Risk Reduction Behaviors Regarding $\mathrm{PM}_{2.5}$ Exposure among Outdoor Exercisers in the Nanjing Metropolitan Area, China. Int J Environ Res Public Health 2018;15:e1728.

16 Zhan $\mathrm{Y}$, Johnson K, Norris C, et al. The influence of air cleaners on indoor particulate matter components and oxidative potential in residential households in Beijing. Sci Total Environ 2018;626:507-18.

17 Lee J-Y, Leem J-H, Kim H-C, et al. Land use regression model for assessing exposure and impacts of air pollutants in school children. Journal of Korean Society for Atmospheric Environment 2012;28:571-80.

18 Clark ML, Butler LM, Koh W-P, et al. Dietary fiber intake modifies the association between secondhand smoke exposure and coronary heart disease mortality among Chinese non-smokers in Singapore. Nutrition 2013;29:1304-9.

19 Saenen ND, Bové H, Steuwe C, et al. Children's urinary environmental carbon load. A novel marker reflecting residential ambient air pollution exposure? Am J Respir Crit Care Med 2017;196:873-81.

20 Yang $\mathrm{H}-\mathrm{J}$, Kim S-H, Jang A-S, et al. Guideline for the prevention and management of particulate matter/yellow dust-induced adverse health effects on the patients with bronchial asthma. $J$ Korean Med Assoc 2015;58:1034-43.

21 Noh J, Sohn J, Cho J, et al. Short-Term effects of ambient air pollution on emergency department visits for asthma: an assessment of effect modification by prior allergic disease history. J Prev Med Public Health 2016;49:329-41.

22 Szyszkowicz M, Kousha T, Castner J, et al. Air pollution and emergency department visits for respiratory diseases: a multi-city case crossover study. Environ Res 2018;163:263-9.

$23 \mathrm{Wu}$ S, Ni Y, Li H, et al. Short-Term exposure to high ambient air pollution increases airway inflammation and respiratory symptoms in chronic obstructive pulmonary disease patients in Beijing, China. Environ Int 2016;94:76-82.

24 Sinharay R, Gong J, Barratt B, et al. Respiratory and cardiovascular responses to walking down a traffic-polluted road compared with walking in a traffic-free area in participants aged 60 years and older with chronic lung or heart disease and age-matched healthy controls: a randomised, crossover study. Lancet 2018;391:339-49.

25 Sack C, Vedal S, Sheppard L, et al. Air pollution and subclinical interstitial lung disease: the multi-ethnic study of atherosclerosis (MESA) air-lung study. Eur Respir J 2017;50:1700559.

26 Wang M, Aaron CP, Madrigano J, et al. Association between longterm exposure to ambient air pollution and change in quantitatively assessed emphysema and lung function. JAMA 2019;322:546-56.

27 Hao Y, Balluz L, Strosnider H, et al. Ozone, fine particulate matter, and chronic lower respiratory disease mortality in the United States. Am J Respir Crit Care Med 2015;192:337-41.

28 Maestas MM, Brook RD, Ziemba RA, et al. Reduction of personal $\mathrm{PM}_{2.5}$ exposure via indoor air filtration systems in Detroit: an intervention study. J Expo Sci Environ Epidemiol 2019;29:484-90. 